\title{
Long-wavelength instability in broad-area semiconductor lasers
}

\author{
F. Prati* and L. Columbo ${ }^{\dagger}$ \\ CNISM and INFM - CNR, Dipartimento di Fisica e Matematica, Università dell'Insubria, via Valleggio 11, I-22100 Como, Italy \\ (Received 9 February 2007; published 14 May 2007)
}

\begin{abstract}
We introduce a set of effective Maxwell-Bloch equations for broad area semiconductor lasers, and study the long-wavelength instability of the homogeneous solution. Unlike in two-level lasers, the presence of the semiconductor $\alpha$ factor allows us to observe this pattern forming instability even in the frequency domain where the homogeneous solution emission has the lower threshold.
\end{abstract}

DOI: 10.1103/PhysRevA.75.053811

PACS number(s): 42.55.Px, 42.60.Mi, 42.65.Sf

\section{INTRODUCTION}

A pattern forming long-wavelength instability for broadarea two-level lasers was discovered in 1988 by Lugiato, Oldano, and Narducci (LON) [1]. They demonstrated that the nonzero homogeneous solution is unstable against any tilted traveling wave with wave-vector $K$ such that

$$
K^{2}<2 \Delta \frac{\mu-\mu_{\mathrm{thr}}}{\mu},
$$

where $\Delta$ is the atomic detuning, $\mu$ is the pump parameter, and $\mu_{\text {thr }}$ is the laser threshold.

Later, it was recognized that the above instability belongs to the class of long-wavelength instabilities that appear in the context of the complex Ginzburg-Landau equation (CGLE) $[2,3]$, and it reduces to the Benjamin-Feir instability when the laser material variables are adiabatically eliminated and the weak-field limit is assumed [4]. At the same time, however, it was pointed out that in a broad area laser the homogeneous solution is the first lasing solution only for $\Delta<0$, and under that condition the LON instability does not exist, as shown by Eq. (1). For $\Delta>0$ the first lasing solution is a tilted traveling wave with wave-vector $\sim \sqrt{\Delta}$, which is affected by other kinds of instability [5]. Hence, the role of the LON instability was confined to the rather unrealistic situation where the laser chooses the homogeneous solution for $\Delta>0$, although the traveling wave has a larger gain.

At the level of envelope equations, the laser dynamics close to threshold can be caught by a CGLE only for $\Delta<0$ [6-8], and in that limit the LON instability does not exist. For $\Delta>0$ the diffusion terms in the CGLE becomes negative, leading to nonphysical antidiffusion. In that case a complex Swift-Hoehnberg equation (CHSE) must be introduced, which accounts for the mechanism that selects at threshold the traveling wave with wave-vector $\sim \sqrt{\Delta}[8-10]$. The LON instability was also analyzed in that framework, but just to show that it is not the dominant pattern forming instability [8].

The above results were obtained for two-level lasers, but sometimes they were applied to the interpretation of the spatiotemporal dynamics of broad-area semiconductor lasers $[11,12]$. In this paper we show that the transposition of the

\footnotetext{
*Electronic address: franco.prati@uninsubria.it
}

†Electronic address: lorenzo.columbo@uninsubria.it results from two-level to semiconductor lasers may be not appropriate, at least for what concerns the LON instability, which in a semiconductor laser plays a much more important role than in a two-level laser.

To that aim we introduce a suitable set of effective Maxwell-Bloch equations (EMBEs) for a broad-area semiconductor laser. These equations are cast in a form as close as possible to the two-level Maxwell-Bloch equations (MBEs) to facilitate the comparison, but with an important difference. While in the MBEs, nonlinear dispersion and the mechanism of wave-vector selection at threshold are both associated with the atomic detuning $\Delta$, in the EMBEs two different parameters appear: the linewidth enhancement factor $\alpha$ for nonlinear dispersion and an effective atomic detuning, that will still be called $\Delta$, for wave-vector selection at threshold.

The stability analysis of the nonzero homogeneous solutions in the EMBEs yields an instability condition which is formally equivalent to Eq. (1), but with the atomic detuning replaced by the linewidth enhancement factor $\alpha$, which means that the instability is due to the combination of diffraction and nonlinear dispersion. This apparently small difference has important consequences. In fact, since $\alpha$ in a semiconductor laser is usually positive, the existence of the long-wavelength instability is no longer confined, as it was in two-level lasers, to the case $\Delta>0$. For $\Delta<0$ the homogeneous solution is the first lasing solution and it is destabilized through the LON instability, which is therefore the main pattern forming mechanism for that side of the detuning. Moreover, the numerical simulations show that even for $\Delta>0$, the traveling wave which is selected at threshold can soon be destabilized in favor of the homogeneous solution as the pump increases, and from that moment on the behavior of the laser is very similar to that observed for $\Delta<0$. Hence, the LON instability plays a fundamental role in triggering the spatiotemporal dynamics of a broad area semiconductor laser for both signs of the detuning.

In Sec. II we derive the EMBEs and discuss the connections with other similar sets of equations proposed in the past for semiconductor lasers. In Sec. III we study analytically and numerically the LON instability in a broad area semiconductor laser in the framework of the EMBEs, and comment on the capability of reduced sets of equation to reproduce the same results. Finally, in Sec. IV we draw the conclusions of our work. 


\section{THE EFFECTIVE MAWELL-BLOCH EQUATIONS}

Two different approaches can be followed to derive EMBEs for semiconductor lasers. The first consists in starting from the microscopic semiconductor Bloch equations and, under determinate assumptions, derive from them two equations for the macroscopic polarization and carrier density variables. From the equation for the macroscopic polarization one can then derive an expression for a two-level-like susceptibility, which, if the simplifying assumptions introduced before are correct, will reproduce well the semiconductor susceptibility calculated at the microscopic level [13]. Alternatively, one can start from a two-level-like susceptibility that establishes a link between the Fourier components of the electric field and of a macroscopic polarization. By Fourier transforming that relation, a dynamical equation for the macroscopic polarization is obtained, which is then coupled with the equations for the electric field and carrier density. The two-level-like susceptibility can be obtained by introducing some assumptions, such as that of zero temperature, in the calculations based on the microscopic model [14]. Another possibility is to make a reasonable guess on the form of the two-level-like susceptibility, which for example can be written as a sum of Lorentzians [15]. If the proposed susceptibility fits well a reference one calculated from first principles, the initial guess is validated.

We follow this pragmatic approach, and assume that the macroscopic susceptibility can be decomposed in the sum of two parts, one depending only on the carrier density $N$ and the other depending also on the frequency $\omega[15]$

$$
\chi(N, \omega)=\chi_{0}(N)+\chi_{1}(N, \omega) .
$$

We write the frequency dependent part as

$$
\chi_{1}(N, \omega)=\frac{\mathcal{A}(N)}{\mathcal{B}(N)-i\left(\omega-\Delta_{0}\right)},
$$

where $\mathcal{A}(N)$ and $\mathcal{B}(N)$ are in general complex functions. The frequency shift $\Delta_{0}$ with respect to the reference frequency $\omega=0$ (the frequency of an empty cavity longitudinal mode) is introduced for later convenience. With respect to [15], Eq. (3) is more general because we allow $\mathcal{A}(N)$ to be a complex function. At this level the only restriction we pose is $\operatorname{Re}(\mathcal{B})$ $>0$ which implies that $\chi_{1}(N, \omega)$ is an analytic function of the complex variable $\omega$ in the upper half plane.

According to our assumptions on the susceptibility $\chi(N, \omega)$, with every component $E(\omega)$ of the electric field envelope is associated a macroscopic polarization

$$
P(N, \omega)=\epsilon_{0} \epsilon_{b} \chi_{0}(N) E(\omega)+P_{1}(N, \omega),
$$

where $\epsilon_{b}$ is the background dielectric constant $\left(n=\sqrt{\epsilon_{b}}\right.$ is the background refractive index), and

$$
P_{1}(N, \omega)=\epsilon_{0} \epsilon_{b} \chi_{1}(N, \omega) E(\omega) .
$$

By Fourier transforming Eq. (5) and neglecting the slow time dependence of $N$ we obtain the dynamical equation for $P_{1}$

$$
\dot{P}_{1}=\epsilon_{0} \epsilon_{b} \mathcal{A}(N) E-\mathcal{B}(N) P_{1}-i \Delta_{0} P_{1},
$$

which must be coupled with the equations for the electric field and the carrier density [15]

$$
\begin{gathered}
\dot{E}=\frac{i \omega_{0} \Gamma_{c}}{2 \epsilon_{0} n n_{g}} P_{1}-\frac{E}{2 \tau_{p}}+\frac{i \omega_{0} n \Gamma_{c} \chi_{0}(N)}{2 n_{g}} E \\
\dot{N}=\frac{I}{q V}-\frac{N}{\tau_{e}}+\frac{1}{4 \hbar i}\left(E^{*} P_{1}-E P_{1}^{*}\right)+\frac{\epsilon_{0} \epsilon_{b}}{4 \hbar i}\left[\chi_{0}(N)-\chi_{0}(N)^{*}\right]|E|^{2}
\end{gathered}
$$

In Eq. (7), $\tau_{p}$ is the photon lifetime, $n_{g}$ is the group index, and $\Gamma_{c}$ is the confinement factor. In Eq. (8), $\tau_{e}$ is the interband carrier relaxation time, $I$ is the injected current, $q$ is the elementary charge, and $V$ is the quantum-well volume.

Equations (7) and (8) are formally equivalent to those derived in [15]. With a suitable rescaling of variables and parameters they can be shown also to be equivalent to those written in [16]. What makes the difference between $[15,16]$, and those models and ours, are the assumptions made on the functions $\chi_{0}(N), \mathcal{A}(N)$, and $\mathcal{B}(N)$. In [15] $\mathcal{A}(N)$ was assumed to be a real function, which implies that the gain curve is a Lorentzian. In [16] it was assumed that $\chi_{0}$ is a complex constant, whose value is determined by the requirement of having no gain in absence of injected carriers. Here, we assume that $\chi_{0}$ is a real constant. One advantage of that choice is straightforward: if $\chi_{0}$ is real the last term in Eq. (8) vanishes. Moreover, the last term in Eq. (7) becomes a simple oscillating term, exactly as the last term in Eq. (6). We can eliminate both setting $\Delta_{0}=-\omega_{0} n \Gamma_{c} \chi_{0} / 2 n_{\mathrm{g}}$ and changing the reference frequency from $\omega=0$ to $\omega=\Delta_{0}$. The new dynamical equations are

$$
\begin{gathered}
\dot{E}=\frac{i \omega_{0} \Gamma_{c}}{2 \epsilon_{0} n n_{g}} P_{1}-\frac{E}{2 \tau_{p}}, \\
\dot{P}_{1}=\epsilon_{0} \epsilon_{b} \mathcal{A}(N) E-\mathcal{B}(N) P_{1}, \\
\dot{N}=\frac{I}{q V}-\frac{N}{\tau_{e}}+\frac{1}{4 \hbar i}\left(E^{*} P_{1}-E P_{1}^{*}\right) .
\end{gathered}
$$

The assumption of $\chi_{0}$ real and constant allows to greatly simplify the EMBEs and write them in a form very close to the MBEs. The price to pay is that the two-level-like susceptibility, which after the change of reference frequency reads

$$
\chi(N, \omega)=\chi_{0}+\frac{\mathcal{A}(N)}{\mathcal{B}(N)-i \omega},
$$

is real for large frequencies, where the contribution of the second term becomes negligible. Hence, it is not able to account for the transition from gain to absorption that occurs normally for large frequencies in real semiconductor materials. However, it must be kept in mind that any set of EMBEs is based on the quasiequilibrium approximation, which limits the validity of the model to a band of width not larger than $\sim 1 \mathrm{THz}$. As we shall see, the approximated susceptibility reproduces well the real one in a band of that width around 


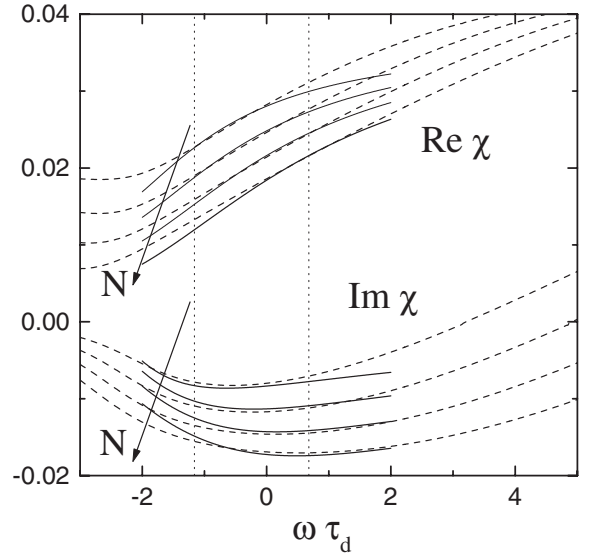

FIG. 1. The real and imaginary parts of the susceptibility calculated with the microscopic theory (dashed lines) for a $6 \mathrm{~nm}$ thick GaAs $/ \mathrm{Al}_{0.2} \mathrm{Ga}_{0.8} \mathrm{As}$ quantum well are represented together with the fitting function $\chi(N, \omega)$ (solid lines). The carrier density $N$ grows in the direction of the arrow, and it takes the values 1.75, 2.0, 2.25, $2.5 \times 10^{24} \mathrm{~m}^{-3}$. The fitting parameters are $f_{0}=1.24 \times 10^{-2}, N_{0}$ $=1.05 \times 10^{24} \mathrm{~m}^{-3}, \quad \Gamma_{0}=-2.87, \quad \Gamma_{1}=2.80, \quad \delta_{0}=-3.09, \quad \delta_{1}=1.51, \quad \alpha$ $=1.08$, and $\chi_{0}=3.71 \times 10^{-2}$.

the maximum gain. What happens for larger frequencies is not relevant.

The EMBEs (9)-(11) formally coincide with those derived in [13], starting from the microscopic semiconductor Bloch equations. However, with respect to [13], we express the functions $\mathcal{A}(N)$ and $\mathcal{B}(N)$ in terms of important parameters such as the linewidth enhancement factor $\alpha$, the transparency carrier density $N_{0}$, the gain width $\Gamma(N)$ and the detuning $\delta(N)$ between the gain peak and the reference frequency. An $N$-independent linewidth factor $\alpha$ calculated at the reference frequency can be introduced assuming that both the real and the imaginary part of $\chi(N, 0)=\chi_{0}+\mathcal{A}(N) / \mathcal{B}(N)$ depend linearly on $N$. If we write

$$
\frac{\mathcal{A}(N)}{\mathcal{B}(N)}=-f_{0}(\alpha+i)\left(\frac{N}{N_{0}}-1\right),
$$

where $f_{0}$ is a real constant, $\alpha$ agrees with the usual definition. The gain width $\Gamma(N)$ and the detuning $\delta(N)$ are defined by

$$
\mathcal{B}(N)=[\Gamma(N)(1-i \alpha)+2 i \delta(N)] / \tau_{d},
$$

where $\tau_{d}$ is the dephasing time of the dipoles. In order for $\Gamma(N)$ and $\delta(N)$ to have the meaning introduced above, it must be $|\delta(N)| \ll \Gamma(N)$. The requirement $\operatorname{Re}(\mathcal{B})>0$ amounts to the condition $\Gamma(N)>0$.

The susceptibility defined by Eqs. (12)-(14) was used to fit a susceptibility calculated with the microscopic theory including many-body effects [17], as shown in Fig. 1. With the additional assumption that $\Gamma(N)$ and $\delta(N)$ vary linearly with $N$ according to $\Gamma(N)=\Gamma_{0}+\Gamma_{1} N / N_{0}$ and $\delta(N)=\delta_{0}$ $+\delta_{1} N / N_{0}$, the fitting parameters are $f_{0}, N_{0}, \Gamma_{0}, \Gamma_{1}, \delta_{0}, \delta_{1}, \alpha$, and $\chi_{0}$. The calculated values are reported in the figure caption. The relative error is smaller than $10 \%$ in a band of frequencies represented by the vertical dotted lines, whose width is close to $2 \tau_{d}^{-1} /(2 \pi)$, that is about $3 \mathrm{THz}$ if $\tau_{d}$ $=100 \mathrm{fs}$.

The final form of the EMBEs, suitable to study the spatiotemporal dynamics of a broad area semiconductor laser are obtained by including diffraction and carrier diffusion by means of the transverse Laplacian $\nabla^{2}$ [18-20], which is defined in terms of transverse coordinates scaled to the diffraction length

$$
\begin{gathered}
\dot{F}=\sigma\left(P-F+i \nabla^{2} F\right), \\
\dot{P}=[\Gamma(D)(1-i \alpha)+2 i \delta(D)][(1-i \alpha) F D-P], \\
\dot{D}=b\left[\mu-D-\frac{1}{2}\left(F^{*} P+F P^{*}\right)+d \nabla^{2} D\right] .
\end{gathered}
$$

The new adimensional variables are related to the old ones by

$$
F=\eta_{1} E e^{i \Delta_{0} t}, \quad P=\eta_{2} P_{1} e^{i \Delta_{0} t}, \quad D=\eta_{3}\left(\frac{N}{N_{0}}-1\right),
$$

with $\eta_{1}^{2}=f_{0} \epsilon_{0} \epsilon_{b} \tau_{e} /\left(2 \hbar N_{0}\right), \quad \eta_{2}=i \omega_{0} \Gamma_{c} \tau_{p} \eta_{1} /\left(\epsilon_{0} n n_{g}\right)$, and $\eta_{3}$ $=f_{0} \omega_{0} \Gamma_{c} \tau_{p} n / n_{g}$. The parameter $\mu=\eta_{3}\left[\left(I \tau_{e}\right) /\left(q V N_{0}\right)-1\right] \mathrm{de}-$ scribes the pump; $d$ is the diffusion coefficient. Time is scaled to $\tau_{d}$, and $\sigma=\tau_{d} /\left(2 \tau_{p}\right), b=\tau_{d} / \tau_{e}$. We assume $\sigma$ $=0.025$ and $b=0.0001$, which means $\tau_{p}=2$ ps and $\tau_{e}=1 \mathrm{~ns}$.

In order to pass from $\Gamma(N)$ and $\delta(N)$ to $\Gamma(D)$ and $\delta(D)$ a value must be assigned to the parameter $\eta_{3}$. This parameter is also related to the ratio $N_{\mathrm{thr}} / N_{0}$ of the threshold to the transparency carrier density. In fact, since at threshold $D$ $\approx 1$, we have $N_{\text {thr }} / N_{0}=1+1 / \eta_{3}$. We chose $\eta_{3}=1$, which means that the threshold carrier density is twice the transparency carrier density. With that choice and with the fit parameters written in the caption of Fig. 1 we obtain $\Gamma(D)=$ $-0.07+2.80 D$ and $\delta(D)=-1.58+1.51 D+\delta^{\prime}$.

The parameter $\delta^{\prime}$ has been introduced to put in evidence that the actual value of $\delta(D)$ depends on the relative position of the reference frequency, which is the empty cavity frequency shifted by the amount $\Delta_{0}$, with respect to the gain peak. If $\delta^{\prime}=0$ the reference frequency $\omega=0$ is placed exactly in between the minima of the two curves of $\operatorname{Im} \chi$ calculated with the smallest and the largest values of $N$, as in Fig. 1. If $\delta^{\prime}$ is positive (negative) the reference frequency is shifted to the dashed (from the solid). This degree of freedom will be used in the next section to study the two situations of positive and negative effective atomic detuning.

Equations (15)-(17) differ from the two-level MBEs only in the equation for $P$, where the multiplicative term $\Gamma(D)$ $\times(1-i \alpha)+2 i \delta(D)$ has been introduced. That term is enough to account for the main features of the semiconductor susceptibility. If $\delta(D)=\alpha \Gamma(D)$ and $\Gamma$ is independent from $D$, the EMBEs are formally equivalent to the two-level MBEs.

With the addition of a term which describes a field injected in the cavity, Eqs. (15)-(17) have been already used to study cavity solitons in a driven VCSEL above threshold [21]. They were particularly useful in that context for two reasons. On one hand, for what concerns the homogeneous 


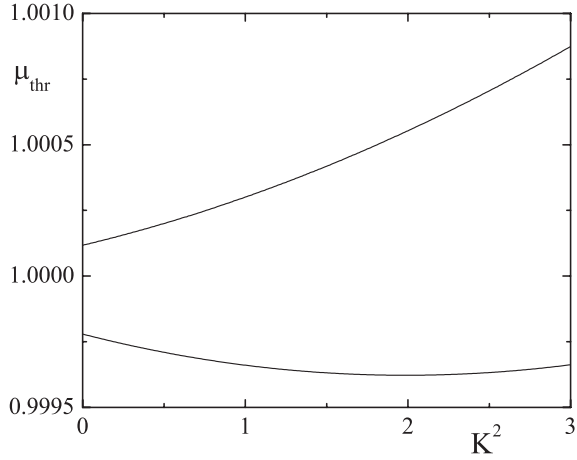

FIG. 2. Laser threshold for $\sigma=0.025, \alpha=1.08$, and $\Gamma(1)=2.73$. The threshold is minimum at $K^{2}=0$ for $\Delta=-2$ (upper curve) and at $K^{2} \simeq \Delta$ for $\Delta=2$ (lower curve)

stationary state and the Turing instability they are equivalent to the rate equations already applied to the VCSEL below threshold, and this allows for a straightforward extension of the results from below to above threshold. On the other hand, since they account for the finite bandwith of the gain, they remove the nonphysical short-wavelength instability that affects the rate equations in the above threshold case below the injection locking point.

\section{THE LON INSTABILITY IN SEMICONDUCTOR LASERS}

The most general stationary solutions of Eqs. (15)-(17) are tilted traveling waves with transverse wave vector $\mathbf{K}$ of the form

$$
E=E_{s} e^{i(\mathbf{K} \cdot \mathbf{x}-\Omega \tau)}, \quad P=P_{s} e^{i(\mathbf{K} \cdot \mathbf{x}-\Omega \tau)}, \quad D=D_{s} .
$$

A useful approximation in the study of these solution and of their stability consists in assuming $\Gamma(D)=\Gamma(1)$ and $\delta(D)$ $=\delta(1)$, which is well justified because $D$ in the stationary state is clamped to the threshold value $D \approx 1$. Moreover, the smallness of $\sigma$ allows to get simple approximated expressions of the relevant quantities. If we write the detuning $\delta(1)$ as

$$
\delta(1)=\sigma(\Delta+\alpha)
$$

with $\Delta$ of order unity, we obtain for the laser threshold $\mu_{\text {thr }}$ and frequency $\Omega$

$$
\begin{gathered}
\mu_{\mathrm{thr}}=1+\sigma^{2} \frac{\left(K^{2}+\alpha\right)\left(K^{2}-\alpha-2 \Delta\right)}{\left(1+\alpha^{2}\right) \Gamma^{2}(1)}+\mathcal{O}\left(\sigma^{3}\right), \\
\Omega=\sigma\left(K^{2}+\alpha\right)+\mathcal{O}\left(\sigma^{2}\right) .
\end{gathered}
$$

The parameter $\Delta$ plays the same role as the atomic detuning in two-level lasers in the selection of the wave vector at threshold. If $\Delta>0$ the threshold is minimum for $K^{2} \simeq \Delta$, and the laser frequency $\Omega$ coincides with the gain peak $\delta(1)$, as expected. If $\Delta<0$ the threshold is minimum for $K=0$. The two behaviors can be seen in Fig. 2, where we plotted $\mu_{\mathrm{thr}}$ as a function of $K^{2}$ for two opposite values of $\Delta$.

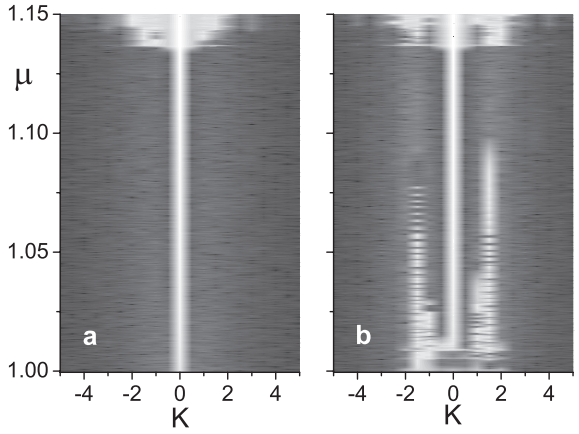

FIG. 3. Intensity spectrum as a function of $\mu$. Same parameters as in Fig. 2, with (a) $\Delta=-2$ and (b) $\Delta=2$. A logarithmic scale is used for the intensity.

The stability analysis of the homogeneous solution $K=0$ reveals that a real eigenvalue is positive if the perturbation is modulated with wave-vector $K$ such that

$$
K^{2}<2 \alpha \frac{\mu-1}{\mu}
$$

which coincides with Eq. (1) with $\alpha$ instead of $\Delta$, and $\mu_{\text {thr }}$ $=1$. Unlike Eq. (1) however, condition (20) is approximated, because it was obtained neglecting terms of order $\sigma$ and carrier diffusion $(d=0)$.

We notice that, according to condition (20), the unstable wave vectors are limited to the band $0<K^{2}<2 \alpha$. In a device of size $L$ the wave vectors take the discrete values $K_{n}$ $=2 \pi n / L, n=0, \pm 1, \pm 2 \ldots$ and the LON instability can be observed only if $K_{1}^{2}<2 \alpha$, which means that $L$ must exceed the minimum value $L_{\min }=\pi \sqrt{2 / \alpha}$. With $\alpha=1.08$ as in Fig. 1, and assuming that the diffraction length, which is the space unit in the model, is $4-5 \mu \mathrm{m}$, we obtain, $L_{\min } \approx 20 \mu \mathrm{m}$. For $L$ $>L_{\min }$ the instability threshold $\mu_{i}$ is

$$
\mu_{i}=\left(1-\frac{K_{1}^{2}}{2 \alpha}\right)^{-1} .
$$

Summarizing, for $\Delta<0$ the homogeneous solution is selected at threshold, but it is stable only up to $\mu_{i}$. In a broad device, where $K_{1}^{2} \ll 2 \alpha$, the stability domain could be very small, and the instability threshold very close to the laser threshold.

This is in sharp contrast with the predictions of MBEs, for which the LON instability does not exist for $\Delta<0$. Instead, the EMBEs agree well with the results of the experiments, where dynamical patterns close to threshold are invariably observed for both signs of the detuning $[11,12,22,23]$. If the MBEs are invoked to explain the experimental findings, the instability of the homogeneous solution cannot be explained for negative detuning. In the past the instability was ascribed to boundary conditions, that alter the nature of the homogenous laser solution, introducing a spatial modulation of the phase [23]. Here we show that the homogeneous solution in a semiconductor laser for $\Delta<0$ is intrinsically unstable, indpendently from the boundary conditions.

In Fig. 3(a) we show the evolution of the intensity spec- 


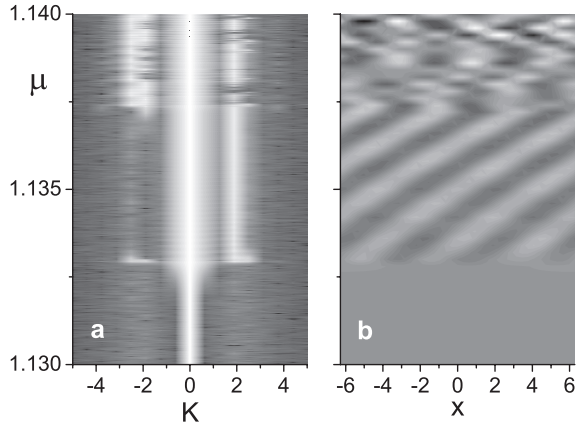

FIG. 4. (a) Intensity spectrum and (b) spatial profile of the intensity in a small region around the LON instability threshold for the same parameters as in Fig. 3(b).

trum (far field) emitted by a one-dimensional device with $K_{1}=0.5(L \approx 50 \mu \mathrm{m})$ and $\Delta=-2$ as the pump is varied continuously from slightly below the laser threshold to $\mu=1.15$ in $15 \times 10^{7}$ time units $(15 \mu \mathrm{s})$. As expected, the homogeneous solution $K=0$ is stable up to the LON instability threshold $\mu_{i}=1.131$. For larger pump values a complex multimode dynamics arise, but the dominant mode is always $K$ $=0$, which means that a low-divergence dynamical pattern is produced. This behavior also agrees with the experimental observations and with the numerical simulations performed using a model similar to that of [15] but which incorporates plasma and lattice heating [24].

The numerical simulations show that the role of the LON instability is not confined to the case $\Delta<0$, where the homogeneous solution is the first lasing solution. Figure 3(b) was obtained with the same parameters as Fig. 3(a), but $\Delta=2$. As expected, the first lasing solution is the traveling wave with $K=-1.5$, which is the one closest to $-\sqrt{\Delta}$. However, this solution becomes unstable almost immediately, and at $\mu$ $=1.1$ the homogeneous solution already prevails. Increasing the pump further, the laser behaves almost exactly as with negative $\Delta$. The fact that for $\Delta>0$ the tilted traveling wave in a semiconductor laser is unstable almost immediately above threshold has been reported also in numerical simulations based on a model where a CSHE for the field is coupled with a mean flow equation for the carrier density [25]. The instability was attributed to the factor $\alpha$ and, in agreement with our simulations, it led to a low divergence turbulent dynamics. A similarity of dynamical behaviors for the two signs of $\Delta$ was also reported in some experiments [12].

To better understand what happens at the LON instability threshold we studied the evolution of the laser in the interval $1.13<\mu<1.14$ varying $\mu$ ten times slower than in Fig. 3, to remove transients. Figure 4(a) shows the intensity spectrum and Fig. 4(b) the corresponding spatial profile of the intensity. These results have been obtained with $\Delta=2$, but they do not differ substantially from those obtained with the opposite value of $\Delta$.

As predicted by the theory, the instability is triggered by the first modes with $K= \pm 0.5$, but their amplitude is so small that no structures can be seen in Fig. 4(b). Soon higher order modes come into play. In the first stage the dominant modes are $K=0$ and $K=1.5$, which give rise to a dynamical pattern with three traveling rolls. A further increase of the pump causes this solution to become unstable and a more complex dynamical pattern appears, where mode $K=-2$ is also important. Hence, although the LON instability is associated with a real eigenvalue, no stationary patterns are observed above $\mu_{i}$.

\section{The LON instability in reduced models}

In two-level lasers the LON instability exists also in the limit in which the material variables are adiabatically eliminated and the model is reduced to a single equation for the complex electric field [1]. One could wonder if the same is true for semiconductor lasers.

In fact, even in the standard rate equation limit of Eqs. (15) -(17) the LON instability can be found. However, those equations, which neglect the frequency dependence of gain, do not describe correctly the multimode dynamics beyond the instability threshold. Moreover they do not account for the mechanism of wave-vector selection at threshold.

A careful analysis of the generalized rate equation model of [26], which include the frequency dependence of gain, would probably reveal that the LON instability is correctly described also within that model. But the parameter $\alpha$ does not appear explicitly there, and this makes less straightforward the derivation of the instability condition. Moreover, in [26] the frequency dependence in the complex susceptibility is introduced through the numerical calculated instantaneous frequency of the electric field, and it is not clear whether such a method allows to follow the complex multimode dynamics exhibited by the laser beyond the instability threshold.

The model of $[25,27]$ consists of a CSHE for the electric field coupled with an equation for the carrier density. As its two-level analogous $[9,10]$, the semiconductor CSHE accounts for wave-vector selection at threshold through an effective atomic detuning parameter. A nonlinear dispersion term proportional to the linewidth enhancement factor $\alpha$ also appears. Hence, the main ingredients present in our EMBEs are contained in that reduced model. Yet, since the model is valid only in the limit of laser very close to threshold, it gives just an approximated instability condition

$$
K^{2}<2 \alpha(\mu-1)
$$

which coincides with Eq. (20) only in the limit $\mu \approx 1$. Correspondingly, the instability threshold according to the CHSE is

$$
\mu_{i, \mathrm{CSHE}}=1+\frac{K_{1}^{2}}{2 \alpha},
$$

and, again, there is a good agreement with Eq. (21) only in the limit of very broad devices, for which $K_{1}^{2} \ll 2 \alpha$ and the instability threshold is very close to the laser threshold. For smaller devices $\mu_{i, \mathrm{CSHE}}$ and $\mu_{i}$ can differ appreciably. Moreover, in the semiconductor CHSE the description of the susceptibility is oversimplified, because the dependence on the carrier density is lost and the gain is symmetric. 


\section{CONCLUSIONS}

In this paper we have shown that the LON instability, discovered in the context of two-level lasers, manifests fully its relevance with semiconductor lasers. For these lasers, in fact, it exists for both signs of the effective atomic detuning $\Delta$. For $\Delta<0$ it is the fundamental mechanism that destabilizes the homogeneous solution which is selected at laser threshold. For $\Delta>0$ numerical simulations have shown that the tilted traveling wave selected at threshold can lose its stability in favor of the homogeneous solution, and then also in that case the transition to complex spatiotemporal dynamics is governed by the LON instability.

The analysis was based on a set of equations which are very close to the MBEs of two-level lasers, but mimic very well the semiconductor susceptibility in a sufficiently wide range of frequencies. We believe that the LON instability and the complex spatiotemporal dynamics that arise from it can be captured by a generalized rate equations model which goes beyond the limitations of the existing ones. The derivation of such a model would allow to extend the numerical analysis to two-dimensional systems for which the complete EMBEs require extremely long computation times.

\section{ACKNOWLEDGMENT}

Work was performed in the framework of the EU-STREP Nr. 4868 FunFACS.
[1] L. A. Lugiato, C. Oldano, and L. M. Narducci, J. Opt. Soc. Am. B 5, 879 (1988).

[2] M. C. Cross and P. C. Hohenberg, Rev. Mod. Phys. 65, 851 (1993).

[3] I. S. Aranson and L. Kramer, Rev. Mod. Phys. 74, 99 (2002).

[4] P. K. Jakobsen, J. V. Moloney, A. C. Newell, and R. Indik, Phys. Rev. A 45, 8129 (1992).

[5] Q. Feng, J. V. Moloney, and A. C. Newell, Phys. Rev. Lett. 71, 1705 (1993).

[6] P. Coullet, L. Gil, and F. Rocca, Opt. Commun. 73, 403 (1989).

[7] G.-L. Oppo, G. D’Alessandro, and W. J. Firth, Phys. Rev. A 44, 4712 (1991).

[8] K. Staliunas, Phys. Rev. A 48, 1573 (1993).

[9] J. Lega, J. V. Moloney, and A. C. Newell, Phys. Rev. Lett. 73, 2978 (1994)

[10] J. Lega, J. V. Moloney, and A. C. Newell, Physica D 83, 478 (1995).

[11] S. P. Hegarty, G. Huyet, J. G. McInerney, and K. D. Choquette, Phys. Rev. Lett. 82, 1434 (1999).

[12] T. Ackemann, S. Barland, M. Cara, S. Balle, J. R. Tredicce, R. Jäger, M. Grabherr, M. Miller, and K. J. Ebeling, J. Opt. B: Quantum Semiclassical Opt. 2, 406 (2000).

[13] J. Yao, G. P. Agrawal, P. Gallion, and C. Bowden, Opt. Commun. 119, 246 (1995).

[14] S. Balle, Opt. Commun. 119, 227 (1995).

[15] C. Z. Ning, R. A. Indik, and J. V. Moloney, IEEE J. Quantum Electron. 33, 1543 (1997).
[16] M. Homar, S. Balle, and M. San Miguel, Opt. Commun. 131, 380 (1996).

[17] W. W. Chow, S. W. Koch, and M. Sargent III, Semiconductor Laser Physics (Springer-Verlag, Berlin, 1994).

[18] Yu. A. Logvin, N. A. Loiko, S. I. Turovets, P. S. Spencer, and K. A. Shore, Laser Phys. 7, 1160 (1997).

[19] C. Z. Ning, J. V. Moloney, A. Egan, and R. A. Indik, Quantum Semiclassic. Opt. 9, 681 (1997).

[20] C. Z. Ning and P. M. Goorjian, J. Opt. Soc. Am. B 16, 2072 (1999).

[21] X. Hachair, F. Pedaci, E. Caboche, S. Barland, M. Giudici, J. R. Tredicce, F. Prati, G. Tissoni, R. Kheradmand, L. A. Lugiato, I. Protsenko, and M. Brambilla, IEEE J. Sel. Top. Quantum Electron. 12, 339 (2006).

[22] S. P. Hegarty, G. Huyet, J. G. McInerney, H. Q. Hou, and K. D. Choquette, in Vertical-Cavity Surface-Emitting Lasers II, edited by K. D. Choquette and R. A. Morgan, Proc. SPIE No. 3286 (SPIE, Bellingham, WA, 1998), pp. 198-204.

[23] S. P. Hegarty, G. Huyet, P. Porta, J. G. McInerney, K. D. Choquette, K. M. Geib, and H. Q. Hou, J. Opt. Soc. Am. B 16, 2060 (1999).

[24] T. Rössler, R. A. Indik, G. K. Harkness, J. V. Moloney, and C. Z. Ning, Phys. Rev. A 58, 3279 (1998).

[25] D. Hochheiser, J. V. Moloney, and J. Lega, Phys. Rev. A 55, R4011 (1997).

[26] S. Balle, Phys. Rev. A 57, 1304 (1998).

[27] J.-F. Mercier and J. V. Moloney, Phys. Rev. E 66, 036221 (2002). 